\title{
Improvised Approach for Real Time Patient Health Monitoring System Using IoT
}

\author{
Parthasarathy $\mathrm{G}^{\mathrm{a}, 1}$, Soumya $\mathrm{T} \mathrm{R}^{\mathrm{b}}$, Ramanathan $\mathrm{L}^{\mathrm{c}}$, and Ramesh $\mathrm{P}^{\mathrm{d}}$ \\ ${ }^{a}$ School of Computing and IT, REVA University, Bangalore \\ ${ }^{b}$ DepT of CSE, Jeppiaar Maamallan Engineering College, Anna University \\ ${ }^{c}$ School of Computer Science and Engineering, VIT University, Vellore \\ ${ }^{b}$ Dept of EEE, CMR Institute of Technology, Bengaluru,560064, Karnataka
}

\begin{abstract}
This paper presents the design details of its implementation of an IoT-based Real Time Health Monitoring System for the monitoring of a patient and provides. The proposed Design permits the monitoring of health related dangers and deals with economy in health care expenditure through effective interpretation and distribution of patient data. This paper works on the concept of reducing human presence in monitoring a patient's problems and sending vital details to the Doctors and Caretakers on a real time basis. This proposal helps saving in and alerts the Doctors/Caretakers in an emergency situation to ensure critical moments are not wasted. The outcome of the proposed system is to provide exact collected medical services to patients through records and information through sensors which monitor the patient's details relating to Pulse, temperature, Body Tilt Angle and Glucose Level. This information is sent to the patient's Doctor/Caretaker using a cloud based App on a real time basis.
\end{abstract}

Keywords. IoT, Patient healthcare, Real Time Health Monitoring System, ATmega328P

\section{Introduction}

Over the last few years, there have been developments of many convincing steps in the field of Internet of Things which has enabled creation of solutions which have led to the introduction of innovative applications. The devices have close links are to each other with numerous smart technologies for the creation of an international ubiquitous network referred to Internet of Things (IoT). Technology improvements coupled with IoT[8][9] have helped generation of facts leading to new methods of utilizing the data. Data generated with the help of the IoT devices have been used for analysis and selection making techniques. The applications of IoT fall into groups related to areas that include transport and logistics, healthcare, smart environment etc. IoT plays a vital role in these areas. Each has assistance from gadgets that get connected with the mobile community, social networks and different smart technologies.

An interesting application of the gadgets is the field of healthcare. A number of technologies can be used to bringing down the overall expenses and help create a stable structure for the management of a patient's ailment. Analysis of the patient's

\footnotetext{
${ }^{1}$ Parthasarathy G, School of Computing and IT, REVA University, Bangalore;

Email : parthasarathy.g@reva.edu.in
} 
health condition[7] requires various remedial parameters. Collection of the parameters and their forwarding to the physician in route the appropriate channel is a challenging job.These technologies work on the basis of devices that do monitoring of indicators in real time on an on-going basis. Increased access to internet and smart phones over the last decade has been witnessing increasing use of internet and smart phones. This has helped close monitoring of the patient's health.

\section{Literature survey}

M. Hassanalieragh et al. [6] have explained in specific, Usage of IoT in the field of healthcare in the developing world. The availability of information beyond comprehensible scales together with innovation generation of artful processing algorithms. (a) Has brought in its train sweeping changes in the scenario of monitoring patient's health a proactive framework for analysis of diseases at the incipient level, tagged on to prevention, cure, and control of fitness rather than on the disease. (b) These include in the area of proactive of medication that would greatly assist speedy and accurate diagnosis and facilitate the determination of the remedy exclusively for the patient and the focus of the options on the specific situations that have application to the patient and help economizing the cost of health care, with simultaneous stress on effectiveness.

Amna Abdullah, et al. [1] have proposed a device known for its use of arduino its core and a software labview that indicates the status. Patient's health data that as include temperature, coronary heart beat charge, muscle tissues, blood pressure, blood glucose stage, and ECG statistics monitored and displayed. The device has been subjected to an exclusive discipline test which has helped the establishment of reliability and accuracy of its findings. Apart from health record details, the device has the ability to make an assessment of the patient's psychological facts with accuracy.

Bhoomika.B.K, et al. [2] have projected the role Internet of factors as a catalyst for the healthcare and finding extensive use in health care applications. Microcontroller finds its place of entry into the numerous sensors together with a temperature sensor and a pulse oximeter sensor in their PICI8F46K22 project. The microcontroller does the work of picking up the sensor data and forwarding it to the community via Wi-Fi, real time tracking of the fitness care parameters for the physician. Access to records can be had with the assistance of a health practitioner. The control has the same relation with a buzzer that alerts the controller on matter relating to variations seen in the sensor output.

Purnima, et al. [3] have designed and developed a reliable, energy green patient monitoring device. Which has the ability to forward parameters of patient expeditiously. Doctors are able to monitor the patient's fitness parameters (temp, heartbeat, ECG, function) quickly..Aminian M, et al. [5] have developed a system in which the affected person could use a hardware fitted with sensors and equipped with android phone application. The sensors get the body temperature and coronary heart reading of the patient. Which are transferred to an android smart smartphone via Bluetooth/Wi-Fi. The System has a cloud database which provides all information about patients health and enables the physician to prescribe the right medicine using this data stored on cloud. 
Sushama Pawar, et al. [4] have engineered a system which offers fitness care of a higher magnitude to humans in their residence. It is an economic and friendly device. The requirement of domestic based health tracking device is extended now days because fitness care fee is exponential growth in the health concern and the expense involved. A significant feature of the system is the use of android smart cell phone in dealing with medical parameters like body temperature, pulse and ECG.

Literature has clear indications the field of health care using IOT showing rapid use of various technologies. The approach towards a real time patient health monitoring[10,11] has not been fully justified. Various drawbacks are seen where the systems are compared to any real time monitoring system. Hence the suggested technique is efficient and reliable in the domain of real time monitoring systems.

\section{Proposed Methodology}

Patient monitor systems in hospitals are known for continuous monitoring of the patient's critical and crucial signs and symptoms. Hardwiring of the sensors to remain by, bedside monitors or PCs becomes imperative. Thus does not stop with connecting these systems to the patient. A paramedical assistant has to do on-going monitoring all the important parameters of the patient through manual maintaining tune of all of his/her information manually. Use of such a technique is not an easy role and might result in confusion and disastrous consequences in the event of a human error.disaster in the case of a human error.

The system can be used for monitoring the patient's heartbeat, glucose level and temperature. But there is something more that is feasible for this system. It detects and alerts the attendant to the doctor when the patient falls down from the bed. The data is monitored continuously. Through the internet helps monitoring the parameters of the patient.

\subsection{Arduino}

Arduino is an open-supply electronics prototyping platform built on bendy, smoothto-use hardware and technology. It is a microcontroller on a circuit board which facilitates to acquire inputs and produce outputs. Arduino/Genuino Uno is a microcontroller board primarily with the ATmega328P forming its bases. It's components include 14 digital input/output pins, 6 analog inputs, a sixteen $\mathrm{MHz}$ quartz crystal, a USB connection, an electricity jack, an ICSP header and a reset button.

\subsection{Temperature sensor}

LM35 series voltages are LM35 temperature sensors known for the inclusion of precision circuit. It's output has a linear association with the Celsius (Centigrade) temperature. The LM35 sensor as a output has an benefit over linear temperature sensors adjusted in ${ }^{\circ}$ Kelvin. The LM35 sensor does not need any outside calibration or trimming to deliver traditional accuracy of $\pm 1 / 4{ }^{\circ} \mathrm{C}$ at room temperature. It Covers a full range of -55 to $+\mathrm{a} 150^{\circ} \mathrm{C}$ temperature. 


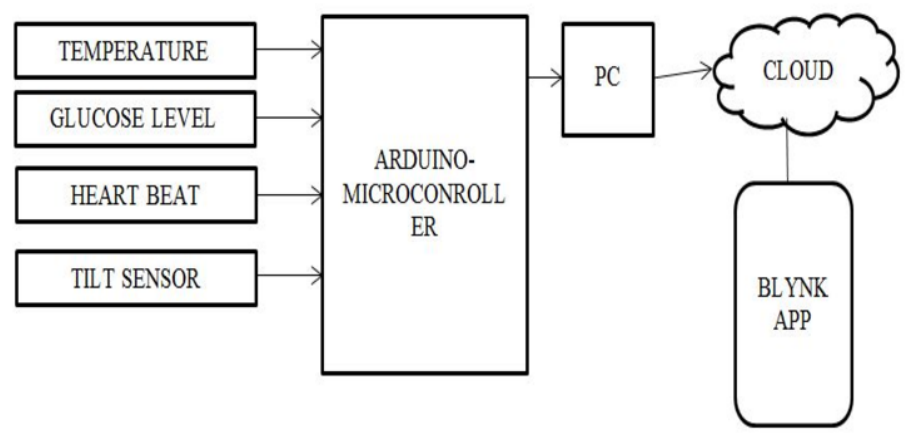

Figure 1. System Architecture

\subsection{Heart beat sensor}

The sensor associated with the heartbeat has, as it based the precept of image photoplethysmography. It does measurement of the variations in the extent of blood through any organ of the body causing an exchange in the light depth through that organ (a vascular location). In case of packages involving the requirement of monitoring coronary heart pulse price, the timing of the pulses assumes considerable significance.. The flow of blood extent is decided by the charge of heart pulses. The light is absorbed via the blood. The signal pulses are equal to the heart beat pulses. There are two types of photoplethysmography:

Transmission: Involves the transmission of the Light emitted from the light emitting tool through any vascular area of the body like earlobe and received via the detector. Reflection: Emission of Light from the light emitting device mirrored through use of the regions.

\subsection{Tilt sensor}

A tilt sensor is an instrument used in the measurement of the lean in two axes of a reference plane. The tilting portion measured is close to gravity. The sensor has the ability to do smooth detection of inclination or orientation. The patient body angle can be indicated taking a single plane and setting with the communication of the fall of the patient following this.

The key specifications of tilt sensors encompass:

- Number of axes: This is an important criterion due to its variations depending on the application.

- Resolution: This is the minimal inclination detected with the help of the sensor.

- Sensitivity: It is a reflection of the sensor's ability to react to small modifications

\subsection{Glucose level sensor}

Float Sensor is an electrical ON/OFF Switch, known for automatic operation when the liquid level goes up or down vis-a-vis to the targeted level. Application of the signal available from the Float Sensor could be useful in the management of the level of the 
glucose administrated to the patient through tubes. A Float Sensor has hermetical closed Reed Control within the stem and a Magnet in the Float. An interesting feature of the float sensor is the trigger by the red switch via the magnet inside the float, increasing or decreasing in tune with the degree of the fluid.

\section{Results and Discussion}

A system has been designed to provide enhanced patient tracking and analysis for the prevention and detection of illness through the tracking of health situations. This is based on the concept of IoT. The results from this system are of great assistance in the investigation of the patient's health condition. The method involves a technique in which the sensor values are received with the assistance from a microcontroller. These values are then uploaded to the cloud using Wi-Fi which is available in a PC. The Patient can view these values in the blynk app on the android or ios device.Blynk is a toolset opensource app for connecting smartphones used in the control of electronics like Arduino, RaspberryPi and similar ones. Remote manipulation of hardware, display of facts by a sensor, ability to save statistics and visualize these is among the exclusive capability factors of Blynk.

The platform has 3 components:

- Blynk App - Used for the creation of the interfaces for use when connected to the microcontroller.

- Blynk Server - known for accessibility to the entire communications between the phone and hardware. Blynk Cloud cab be used or alternatively the Blynk server can have a private run.

- Blynk Libraries - They find use in permitting verbal exchange with the server for the entire range of the incoming and outgoing instructions.

The system is capable of the following results.

- Monitoring of the patient heartbeat, glucose level, temperature precisely.

- Providing communication of the patient falling down from the bed.

- Continuous monitoring of parameters through the Blynk Application

- Forwarding of communication to the physician about any abnormal condition that occurs based on the parameters.

\section{Conclusion}

IoT (Internet of Things) is an innovative automation and analytics system which has exclusive networking, sensing, capabilities relating to providing information of a large manitude, and artificial intelligence technology in the delivery of complete systems for a product or services. These structures permit and feasible transparency of a high degree, control, and overall performance when applied in any industry or to a machine. IoT systems have programs across industries due to their precise flexibility and capacity to be appropriate in any ambience.The various relevant parameters are evaluated and constantly monitored with the help of IOT and complete data is obtained with the assistance of Blynk app. Monitoring of the heartbeat, temperature, glucose stage and Body Tilt Angle of a patient is done and in the event of the happening of any abnormal circumstance, communication is sent to the physician or 
surgeon to enable taking up appropriate remedial action. Remote monitoring of the heartbeat, glucose stage and temperature of the affected person is done using the blynk software through the android/IOS cellular telephone. The tilt sensor is used to communicate the fall of the patient from bed and the doctor can send a remote alert to the nurse within the sanatorium without any delay check of the patient. This device can be coupled with numerous other sensors primarily based on the requirements of finding the physical status to enhance the system functionalities.

\section{References}

[1] Amna Abdullah, Asma Ismael, Aisha Rashid, Ali Abou-ElNour, and Mohammed Tarique. Real time wireless health monitoring application using mobile devices .International Journal of Computer Networks \& Communications IJCNC May 2015;Vol.7, No.3.

[2] Bhoomika.B.K, Dr. K N Muralidharal. Secured Smart Healthcare Monitoring System Based on IoT. International Journal on Recent and Innovation Trends in Computing and Communication Volume: 3 Issue: 7.

[3] Purnima, Puneet Singh,.Zigbee and GSM Based Patient Health Monitoring System. 2014 International Conference on Electronics and Communication System (ICECS-2014).

[4] Sushama Pawar, P.W.Kulkarni. Home Based Health Monitoring System Using Android Smartphone. International Journal of Electical, Electronics and Data Communication, feb 2014;Vol-2, Issue-2 .

[5] Aminian M, Naji HR .A Hospital Healthcare Monitoring System Using Wireless Sensor Networks. J Health Med Inform 4: 121. doi:10.4172/2157-7420.1000121,2013.

[6] M. Hassanalieragh et al., Health Monitoring and Management Using Internet-of-Things (IoT) Sensing with Cloud-Based Processing: Opportunities and Challenges .2015; IEEE International Conference on Services Computing, New York, NY, 2015, p. 285-292.

[7] Boyi Xu, Li Da Xu, Hongming Cai, Cheng Xie, Jingyuan Hu, and Fenglin Bu. Ubiquitous Data Accessing Method in IoT-Based Information System for Emergency Medical Services.IEEE Transactions on Industrial Informatics, May 2014; Vol. 10, No. 2 .

[8] Gennaro Tartarisco, Giovanni Baldus, Dan z Corda, Rossella Raso, Antonino Arnao, Marcello Ferro, Andrea Gaggioli, Giovanni Pioggia .Personal Health System architecture for stress monitoring and support to clinical decisions, Computer Communications ;2012; Vol.35, p.1296-1305 .

[9] VD Ambeth Kumar ; Malathi Subramanian ; Gokul Gopalakrishnan ; Krishnasamy Vengatesan ; Durai Elangovan ; Balakrishnan Chitra . Implementation of the pulse rhythmic rate for the efficient diagnosing of the heartbeat.IET Healthcare Technology Letters,2019; Volume 6, Issue 2,p. 48 - 52.

[10] Virone, G., A. Wood, L. Selavo, Q. Cao, L. Fang, T. Doan, Z. He, and J. Stankovic .An advanced wireless sensor network for health monitoring. In Transdisciplinary Conference on Distributed Diagnosis and Home Healthcare (D2H2), p. 2-4. 2006.

[11] S.Hema Kumar, J.Uday Kiran, V.D.Ambeth Kumar, G.Saranya, Ramalakshmi V, "Effective Online Medical Appointment System", International Journal of Scientific \& Technology Research, Volume 8, Issue 09, September 2019, Pages $803-805$. 